\title{
Correction to: Rational social and political polarization
}

\author{
Daniel J. Singer ${ }^{1}$ - Aaron Bramson ${ }^{2,3,4}$. \\ Patrick Grim $^{5,6}$ • Bennett Holman ${ }^{7}$ Jiin Jung ${ }^{8}$ • \\ Karen Kovaka' ${ }^{9}$ Anika Ranginani ${ }^{1}$. \\ William J. Berger ${ }^{1}$
}

Published online: 11 July 2018

(C) Springer Nature B.V. 2018

\section{Correction to: Philos Stud https://doi.org/10.1007/s11098-018-1124-5}

In the original publication of the article, the Acknowledgement section was inadvertently not included. The Acknowledgement is given in this Correction.

"We are grateful to many folks for their helpful feedback on earlier versions of this work, especially Gregor Betz, Steven Kimbrough, Christof Weinhardt, and audiences at the Karlsruhe Institute of Technology, the University of Pennsylvania, and the University of Michigan."

The original article can be found online at https://doi.org/10.1007/s11098-018-1124-5.

Daniel J. Singer

singerd@phil.upenn.edu

1 University of Pennsylvania, Philadelphia, PA, USA

2 Riken Brain Science Institute, Wakoshi, Japan

3 Ghent University, Ghent, Belgium

4 University of North Carolina at Charlotte, Charlotte, NC, USA

5 University of Michigan, Ann Arbor, MI, USA

6 Stony Brook University, Stony Brook, NY, USA

7 Underwood International College, Yonsei University, Seoul, South Korea

8 Claremont Graduate University, Claremont, CA, USA

9 Virginia Polytechnic Institute and State University, Blacksburg, VA, USA 